\title{
Meet, Discuss, and Segregate!
}

\author{
GÉRARD WEISBUCH, ${ }^{1}$ GUILLAUME DEFFUANT, ${ }^{2}$ FRÉdÉRIC AMBLARD, ${ }^{2}$ JEAN-PIERRE NADAL ${ }^{1}$ \\ ${ }^{1}$ Laboratoire de Physique Statistique, * de l'Ecole Normale Supérieure, 24, rue Lhomond, F-75231 Paris Cedex \\ 5, France; and ${ }^{2}$ Laboratoire d'Ingénierie pour les Systèmes Complexes (LISC), Cemagref, Grpt de \\ Clermont-Ferrand 24, avenue des Landais, BP50085, F-63172 Aubière Cedex, France
}

Received December 4, 2001; revised March 18, 2002; accepted March 18, 2002

\begin{abstract}
We present a model of opinion dynamics in which agents adjust continuous opinions as a result of random binary encounters whenever their difference in opinion is below a given threshold. High thresholds yield convergence of opinions toward an average opinion, whereas low thresholds result in several opinion clusters. The model is further generalized to network interactions, threshold heterogeneity, adaptive thresholds, and binary strings of opinions. (C) 2002 Wiley Periodicals, Inc.
\end{abstract}

Key Words: opinion dynamics, heterogeneous agents, bounded confidence

I he present work was initially motivated by an empirical study about the diffusion of environmentally friendly practices (agri-environmental measures) among European farmers [1]. From 1992 Common Agricultural Policy (CAP) of European Communities was changed from the postwar policy of financially supporting production to a financial support of environmentally friendly practices such as input (fertilizers and pesticide) reduction, setaside, and preservation of biodiversity. The next problem was the implementation by farmers of the policy defined at the highest level, the European Commission. Our "IMAGES" teams then studied the factors and processes, which favor (or prevent) the adoption by farmers of the new policy. The empirical data collected through surveys and interviews

*Laboratoire associé au CNRS (URA 1306), á l'ENS et aux Universités Paris 6 et Paris 7.

Corresponding author: Gérard Weisbuch (e-mail: weisbuch@ lps.ens.fr) with farmers and agricultural counselors are in accordance with previous literature on the Sociology of Agriculture [2] and demonstrate that:

- changing practices involves a lot of uncertainties for the farmer; for instance, fertilizers reduction combined with best farming practices might imply a complete reorganization of crop choice and annual rotation.

- To decrease uncertainties, farmers engage in many discussions with their peers; in addition to the propagation of information, the interactions between farmers perform a normative control and a prestige allocation. The normative control is based on an agreement about what constitutes 'good' or 'bad' farming practices.

- The specific interviews done in our project showed that the decision of adopting an agri-environmental measure is the result of a process, which involves social and informational influences, sometimes during several years (for more information about the interviews and data see Deffuant [3]). 
In this context of numerous information exchanges before any adoption decision, modeling adoption dynamics through methods inspired from information contagion is more appropriate than game theory often used in environmental economics. In the game theoretical approach, payoffs are directly learned by switching behaviors, but the long-term costs of switches are underestimated with respect the "inertial" conditions faced by farmers in Europe: several-year contracts have to be signed with local authority, and they involve major reorganization of the farm (capital investment, crop rotation, labor).

A first possibility to model the information diffusion process is to use binary state models (the state representing whether the farmer adopted green practices or not). Such models correspond to the classical threshold models of innovation diffusion developed by sociologists $[4,5]$. These models assume that each individual has a threshold, which is interpreted as his personal interest to change his behavior. Then, the social pressure is represented as a function of the number of neighbors who already adopted. The decision of adoption is based on the sum of the threshold and this social pressure.

Many models about opinion dynamics [6-10] are based on binary opinions, which social actors update as a result of social influence, often according to some version of a majority rule. Binary opinion dynamics have been well studied, such as the herd behavior described by economists $[6,7,11]$. One expects that the attractor of the dynamics will display uniformity of opinions, either 0 or 1 , when interactions occur across the whole population. Clusters of opposite opinions appear when the dynamics occur on a social network with exchanges restricted to connected agents. Clustering is reinforced when agent diversity, such as a disparity in influence, is introduced [10,12].

One issue of interest concerns the importance of the binary assumption: what would happen if opinion were a continuous variable such as the worthiness of a choice (a utility in economics), or some belief about the adjustment of a control parameter?

The rationale for binary versus continuous opinions is related to the kind of information used by agents to validate their own choice:

- the actual choice of the other agents, a situation common in economic choice of brands: "do as the others do";

- or the actual opinion of the other agents, about the "value" of a choice: "establish one's opinion according to what the others think or at least according to what they say."

More generally, we expect opinion (rather than choice) dynamics to occur in situation where agents have to make important choices and care to collect many opinions before taking any decisions: adopting a technological change might often be the case. Political elections also belong to the same category, because of uncertainties concerning new candidates, new challenges in the future, long electoral mandate (especially in Europe), etc. European integration is an obvious example of a quasi-irreversible decision, which involved many uncertainties and was subject to all sorts of discussions among citizens.

Modeling of continuous opinions dynamics was earlier started by applied mathematicians and focused on the conditions under which a panel of experts would reach a consensus [13-18].

The purpose of this article is to present results concerning continuous opinion dynamics subject to the constraint that convergent opinion adjustment only proceeds when opinion difference is below a given threshold. The rationale for the threshold condition is that agents only interact when their opinions are already close enough; otherwise they do not even bother to discuss. The reason for refusing discussion might be for instance lack of understanding, conflicting interest, or social pressure. The threshold would then correspond to some openness character. Another interpretation is that the threshold corresponds to uncertainty: the agents have some initial views with some degree of uncertainty and would not care about other views outside their uncertainty range.

Social Psychology literature discusses social influence and the conditions under which individual attitudes and decisions are influenced by others (see e.g., Petty and Cacioppo [19]). Part of this literature concentrates on how initial attitudes determine the outcome of interactions [20-22]. One can summarize the general outcome of the reported experiments as an increase of influence when initial positions are close enough. The threshold condition that we introduce here is also used in Axelrod's model of disseminating culture [23].

Many variants of the basic idea can be proposed, and the article is organized as follows:

- We first expose the simple case of complete mixing among agents under a unique and constant threshold condition.

- We then check the genericity of the results obtained for the simplest model to other cases such as localized interactions, distribution of thresholds, varying thresholds, and binary strings of opinions.

A previous publication [24] and a working paper [25] report more complete results on several aspects.

\section{THE BASIC CASE: COMPLETE MIXING AND ONE FIXED THRESHOLD}

Let us consider a population of $N$ agents $i$ with continuous opinion $x_{i}$. We start from an initial distribution of opinions, most often taken uniform on $[0,1]$ in the computer simula- 
tions. At each time step any two randomly chosen agents meet: they readjust their opinion when their difference in opinion is smaller in magnitude than a threshold $d$. Suppose that the two agents have opinion $x$ and $x^{\prime}$. Iff $\left|x-x^{\prime}\right|<d$ opinions are adjusted according to

$$
\begin{gathered}
x=x+\mu \cdot\left(x^{\prime}-x\right) \\
x^{\prime}=x^{\prime}+\mu \cdot\left(x-x^{\prime}\right)
\end{gathered}
$$

where $\mu$ is the convergence parameter whose values may range from 0 to 0.5 .

In the basic model, the threshold $d$ is taken as constant in time and across the whole population. Note that we here apply a complete mixing hypothesis plus a random serial iteration mode. (The "consensus" literature most often uses parallel iteration mode when they suppose that agents average at each time step the opinions of their neighborhood. Their implicit rationale for parallel iteration is that they model successive meetings among experts.)

The evolution of opinions may be mathematically predicted in the limiting case of small values of $d$ [26]. (The other extreme is the absence of any threshold, which yields consensus at infinite time as earlier studied in Stone [13] and others.) For finite thresholds, computer simulations show that the distribution of opinions evolves at large times toward clusters of homogeneous opinions.

- For large threshold values $(d>0.3)$ only one cluster is observed at the average initial opinion. Figure 1 represents the time evolution of opinions starting from a uniform distribution of opinions.

- For lower threshold values, several clusters can be observed (see Figure 2). Consensus is then not achieved when thresholds are low enough.

\section{FIGURE 1}

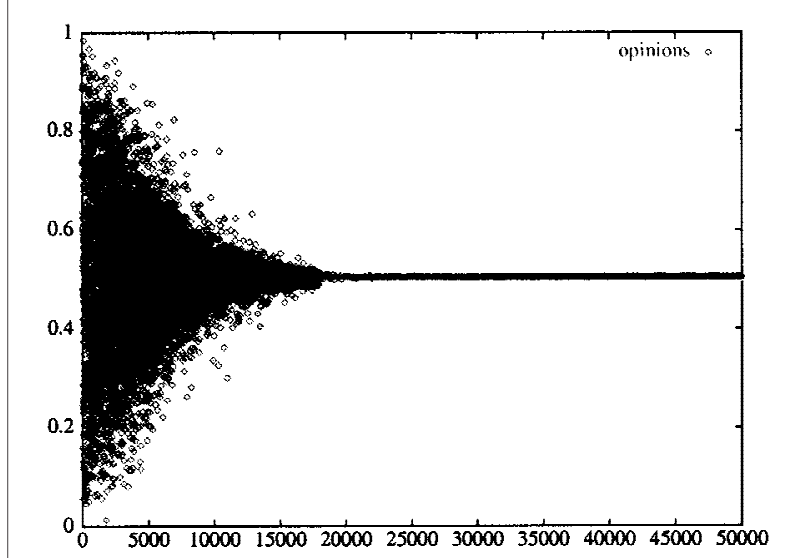

Time chart of opinions $(d=0.5 \mu=0.5 N=2000)$. One time unit corresponds to sampling a pair of agents.

\section{FIGURE 2}

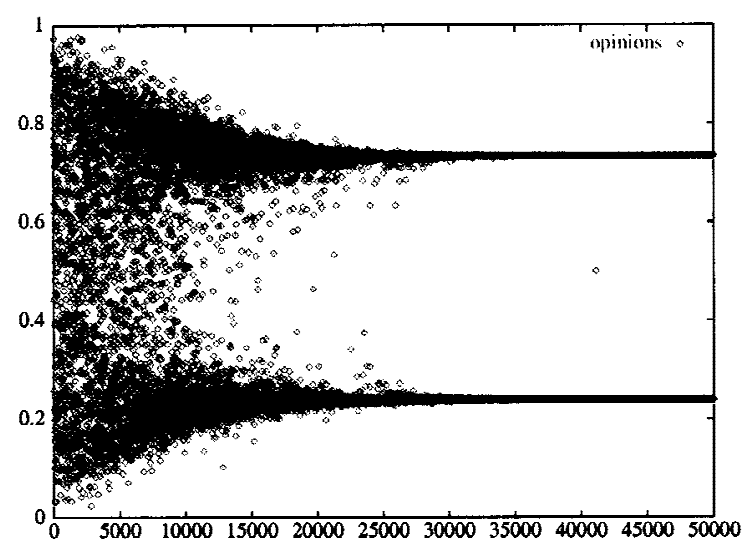

Time chart of opinions for a lower threshold $d=0.2(\mu=0.5, N$ $=1000$ )

Obtaining clusters of different opinions does not surprise an observer of human societies, but this result was not a priori obvious because we started from an initial configuration where transitivity of opinion propagation was possible through the entire population: any two agents however different in opinions could have been related through a chain of agents with closer opinions. The dynamics that we describe ended up in gathering opinions in clusters on the one hand, but also in separating the clusters in such a way that agents in different clusters do not exchange anymore.

The number of clusters varies as the integer part of $1 / 2 \mathrm{~d}$ : this is to be further referred to as the " $1 / 2 \mathrm{~d}$ rule" (see Figure 3 ; notice the continuous transitions in the average number of clusters when $d$ varies. Because of the randomness of the initial distribution and pair sampling, any prediction on the

\section{FIGURE 3}

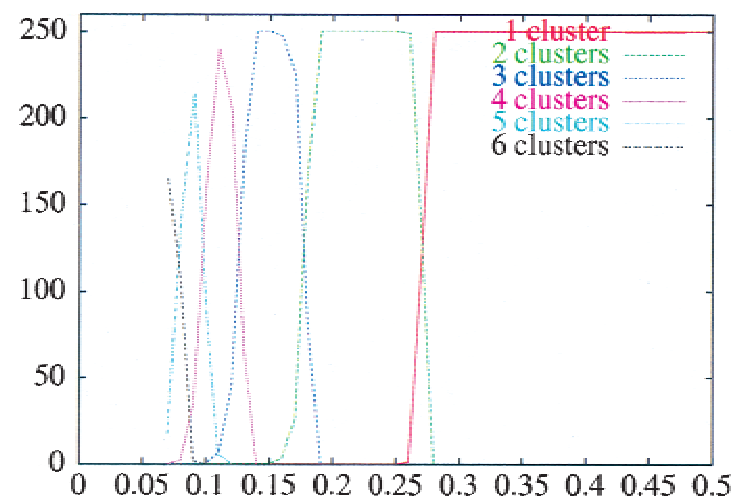

Statistics of the number of opinion clusters as a function of $d$ on the $x$-axis for 250 samples $(\mu=0.5, N=1000)$. 
outcome of dynamics such as the $1 / 2 \mathrm{~d}$ rule can be expressed as true with a probability close to one in the limit of large $N$, but one can often generate a deterministic sequence of updates which would contradict the "most likely" prediction.).

\section{SOCIAL NETWORKS}

The literature on social influence and social choice also considers the case when interactions occur along social connections between agents [6] rather than randomly across the whole population. Apart from the similarity condition, we now add to our model a condition on proximity, i.e., agents only interact if they are directly connected through a pre-existing social relation. Although one might certainly consider the possibility that opinions on certain unsignificant subjects could be influenced by complete strangers, we expect important decisions to be influenced by advice taken either from professionals (doctors, for instance) or from socially connected persons (e.g., through family, business, or clubs). Facing the difficulty of inventing a credible instance of a social network as in the literature on social binary choice, we here adopted the standard simplification and carried out our simulations on square lattices: square lattices are simple, allow easy visualization of opinion configurations, and contain many short loops, a property that they share with real social networks.

We then started from a two-dimensional (2D) network of connected agents on a square grid. Any agent can only interact with his four connected neighbors (N, S, E, and W). We used the same initial random sampling of opinions from 0 to 1 and the same basic interaction process between agents as in the previous sections. At each time step a pair is randomly selected among connected agents, and opinions are updated according to Equations 1 and 2 provided of course that their distance is less than $d$.

The results are not very different from those observed with nonlocal opinion mixing as described in the previous section, at least for the larger values of $d(d>0.3$, all results displayed in this section are equilibrium results at large times).

As seen in Figure 4, the lattice is filled with a large majority of agents who have reached consensus around $x=0.5$, whereas a few isolated agents have "extremists" opinions closer to 0 or 1 . The importance of extremists is the most noticeable difference with the full mixing case described in the previous section.

Interesting differences are noticeable for the smaller values of $d<0.3$ as observed in Figure 5 .

For connectivity 4 on a square lattice, only one cluster percolates [27] across the lattice. All agents of the percolating cluster share the same opinion as observed on Figure 4, but for $d<0.3$ several opinion clusters are observed and none percolates across the lattice.

\section{FIGURE 4}

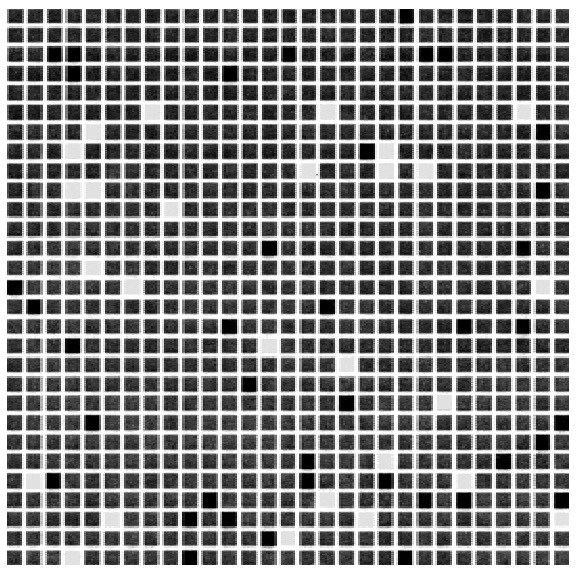

Display of final opinions of agents connected on a square lattice of size $29 \times 29$ ( $d=0.3 \mu=0.3$ after 100,000 iterations). Opinions between 0 and 1 are coded by gray level ( 0 is black and 1 is white). Note the percolation of the large cluster of homogeneous opinion and the presence of isolated "extremists."

Similar opinions, but not identical, are shared across several clusters. The differences of opinions between groups of clusters relate to $d$, but the actual values inside a group of clusters fluctuate from cluster to cluster because homogenization occurred independently among the different clusters: the resulting opinions depend on fluctuations of initial opinions and histories from one cluster to the other. The same increase in fluctuations compared to the full mixing

\section{FIGURE 5}

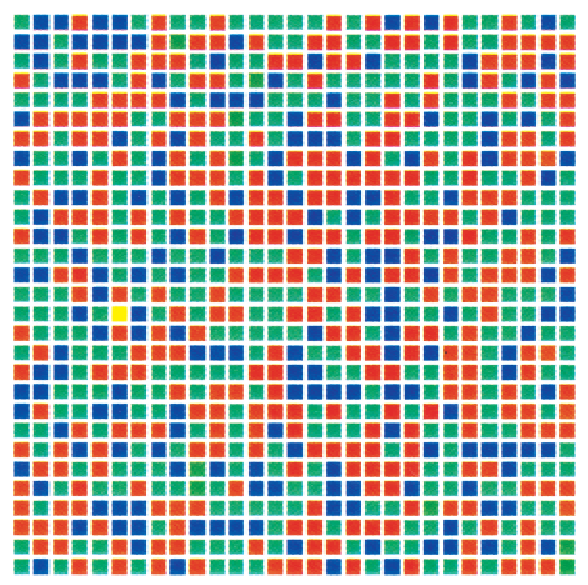

Display of final opinions of agents connected on a square lattice of size $29 \times 29(d=0.15 \mu=0.3$ after 100.000 iterations). Color code: purple 0.14 , light blue 0.42 , red $0.81-0.87$. Note the presence of smaller clusters with similar but not identical opinions. 
case is observed from sample to sample with the same parameter values.

The qualitative results obtained with 2D lattices should be observed with any connectivity, either periodic, random, or small world.

The above results were obtained when all agents had the same invariant threshold. The purpose of the following sections is to check the general character of our conclusions:

- when one introduces a distribution of thresholds in the population;

- when the thresholds themselves obey some dynamics.

\section{HETEROGENEOUS CONSTANT THRESHOLD}

Supposing that all agents use the same threshold to decide whether to take into account the views of other agents is a simplifying assumption. When heterogeneity of thresholds is introduced, some new features appear. To simplify the matter, let us exemplify the issue in the case of a bimodal distribution of thresholds, for instance, 8 agents with a large threshold of 0.4 and 192 with a narrow threshold of 0.2 as in Figure 6.

One observes that in the long run, convergence of opinions into one single cluster is achieved because of the presence of the few "open minded" agents (the single cluster convergence time is 12,000 , corresponding to 60 iterations per agent on average, for the parameters of Figure 6). However, in the short run, a metastable situation with two large opinion clusters close to opinions 0.35 and 0.75 is observed because of narrow minded agents, with open minded agents opinions fluctuating around 0.5 because of interactions with narrow minded agents belonging to either high or low opinion cluster. Because of the few exchanges with the high $d$ agents, low $d$ agents opinions slowly shift toward the

\section{FIGURE 6}

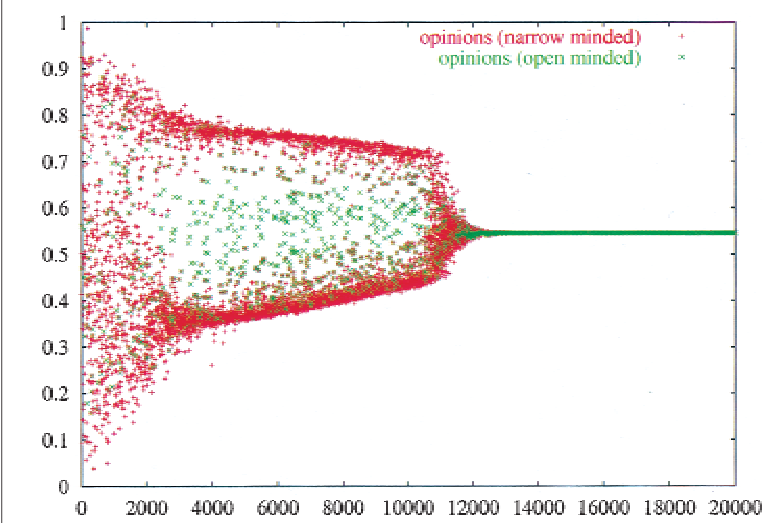

Time chart of opinions $(N=200)$. Red + represent narrow-minded opinions (192 agents with threshold 0.2), green $\times$ represent openminded opinions (8 agents with threshold 0.4). average until the difference in opinions between the two clusters falls below the low threshold: at this point the two clusters collapse.

This behavior is generic for any mixtures of thresholds. At any time scale, the number of clusters obeys a "generalized $1 / 2 \mathrm{~d}$ rule":

- on the long run clustering depends on the higher threshold;

- on the short run clustering depends on the lower threshold;

- the transition time between the two dynamics is proportional to the total number of agents and to the ratio of narrow minded to open minded agents.

In some sense, the existence of a few "open minded" agents seems sufficient to ensure consensus after a large enough time for convergence. The next section restricts the validity of this prediction when threshold dynamics are themselves taken into account.

\section{THRESHOLD DYNAMICS}

\subsection{The Model}

Let us interpret the basic threshold rule in terms of agent's uncertainty: agents take into account others' opinion on the occasion of interaction because they are not certain about the worthiness of a choice. They engage in interaction only with those agents which opinion does not differ too much from their own opinion in proportion of their own uncertainty. If we interpret the threshold for exchange as the agent uncertainty, we might suppose with some rationale that his subjective uncertainty decreases with the number of opinion exchanges.

Taking opinions from other agents can be interpreted, at least by the agent himself, as sampling a distribution of opinions. As a result of this sampling, agents should update their new opinion by averaging over their previous opinion and the sampled external opinion and update the variance of the opinion distribution accordingly.

Within this interpretation, a "rational procedure" (in the sense of Herbert Simon) for the agent is to simultaneously update his opinion and his subjective uncertainty. Let us write opinion updating as weighting one's previous opinion $x(t-1)$ by $\alpha$ and the other agent's opinion $x^{\prime}(t-1)$ by $1-$ $\alpha$, with $0<\alpha<1$. $\alpha$ is a "confidence" parameter weighting how much the agent trust his own opinion with respect to those of others. $\alpha$ can be rewritten $\alpha=1-(1 / n)$, where $n$ expresses a characteristic number of opinions taken into account in the averaging process. $n-1$ is then a relative weight of the agent previous opinion as compared to the newly sampled opinion weighted 1 . Within this interpretation, updates of both opinion $x$ and variance $v$ should be written:

$$
\begin{aligned}
& x(t)=\alpha \cdot x(t-1)+(1-\alpha) \cdot x^{\prime}(t-1) \\
& v(t)=\alpha \cdot v(t-1)+\alpha \cdot(1-\alpha) \cdot\left[x(t-1)-x^{\prime}(t-1)\right]^{2} .
\end{aligned}
$$


The second equation simply represents the change in variance when the number of samples increases from $n-1$ at time $t-1$ to $n$ at time $t$. It is directly obtained from the definition of variance as a weighted sum of squared deviations.

As previously, updating occurs when the difference in opinion is lesser than a threshold, but this threshold is now related to the variance of the distribution of opinions sampled by the agent. A simple choice is to relate the threshold to the standard deviation $\sigma(t)$ according to:

$$
d(t)=\nu \sigma(t)
$$

where $v$ is a constant parameter often taken equal to 1 in the simulations.

When an agent equally values collected opinions independently of how old they are, he should also update his confidence parameter $\alpha$ in connection with $n(t)-1$ the number of previously collected opinions (This expression is also used in the literature about "consensus" building to describe "hardening" of agents' opinions as in Chatterjee and Seneta [14] and Cohen et al. in 1986 [15].):

$$
\alpha(t)=\frac{n(t)-1}{n(t)} \cdot \alpha(t-1) .
$$

Another possible updating choice is to maintain $\alpha$ constant, which corresponds to taking a moving average on opinions and giving more importance to the $n$ later collected opinions. Such a "bounded" memory would make sense in the case when the agent believes that there exists some slow shift in the distribution of opinions, whatever its cause, and that older opinions should be discarded.

Both algorithms were tried in the simulations and give qualitatively similar results in terms of the number of attractors, provided that one starts from an initial number of supposed trials $n(0)$ corresponding to the same $\alpha$. The only difference concerns the dynamics of convergence:

- In the case of constant confidence $\alpha$, convergence is exponential: thresholds, variances, and distances to attractors decay exponentially versus the number of updates experienced by the agents.

- In the case of adjustable confidence $\alpha$, convergence is hyperbolic: variances and distances to attractors decay as the inverse number of updates (thresholds vary as the inverse square root of this number).

These scaling are predicted using simple approximations and verified by simulation.

\subsection{SIMULATION RESULTS}

When compared with constant threshold dynamics, decreasing thresholds results in a larger variety of final opin- ions. For initial thresholds values, which would have ended in opinion consensus, one observes a number of final clusters, which decreases with $\alpha$ (and thus with $n$ ).

Observing the chart of final opinions versus initial opinions on Figure 7, one sees that most opinions converge toward two clusters (at $x=0.60$ and $x=0.42$ ), which are closer than those one would obtain with constant thresholds (typically around $x=0.66$ and $x=0.33$ ): initial convergence gathered opinions which would have aggregated at the initial threshold values (0.5), but which later segregated because of the decrease in thresholds. Many outliers are apparent on the plot.

Large values of $\alpha$, close to one, e.g., $n>7$, correspond to averaging on many interactions. The interpretation of large $\alpha$ and $n$ is that the agent has more confidence in his own opinion than in the opinion of the other agent with whom he is interacting, in proportion with $n-1$. For constant values of $\alpha$, the observed dynamics is not very different from what we obtained with constant thresholds (Figure 8).

A more complicated dynamics is observed for lower values of $n$ and $\alpha$ which correspond to a fast decrease of the thresholds, thus preventing the aggregation of all opinions into large clusters. Apart from the main clusters, one also observes smaller clusters plus outliers (already present on Figure 7).

For $d(0)=0.5$ (which would yield consensus with only one cluster if kept constant) and $\alpha=0.5$ (corresponding to $n$ $=2$, i.e., agents giving equal weight to their own opinion and

\section{FIGURE 7}

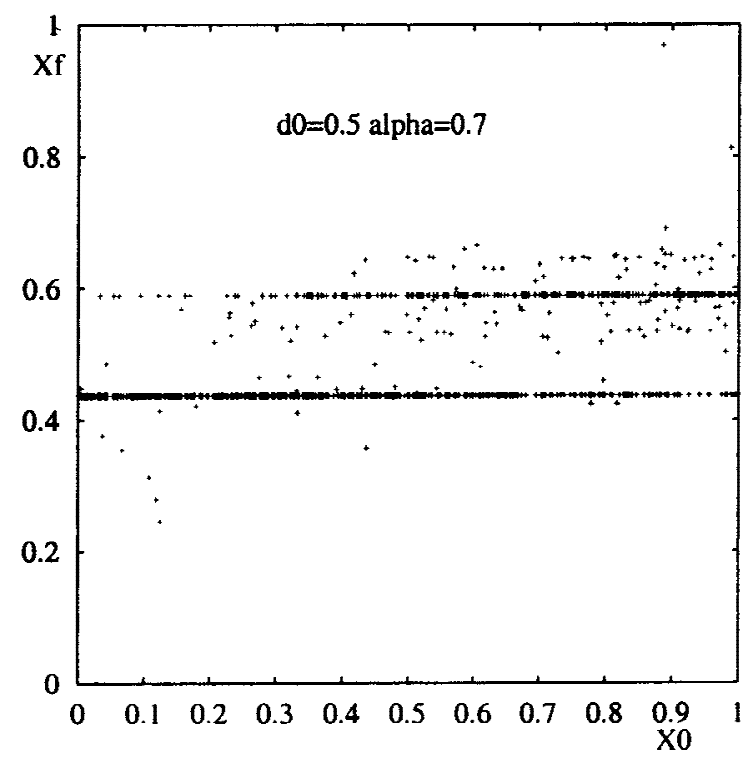

Each point on this chart represents the final opinion of one agent versus its initial opinion (for constant $\alpha=0.7 v=1.0 \mathrm{~N}=1000$, initial threshold 0.5). 


\section{FIGURE 8}

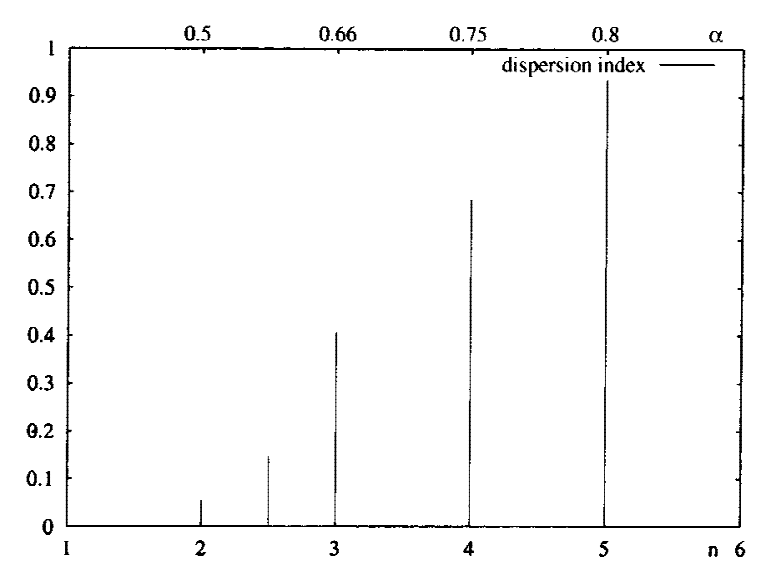

Variation of the dispersion index $y$ with $n$, the initial "subjective" number of collected opinions $(\alpha=1-1 / n, d(0)=0.5 v=1.0 \mathrm{~N}$ $=1000$ ). Small values of $y$ correspond to several attractors, larger values close to one to a single attractor. The initial threshold value of 0.5 if kept constant would yield consensus with only one cluster.

to the external opinion), more than 10 clusters unequal in size are observed plus isolated outliers. One way to characterize the dispersion of opinions with varying $\alpha$ is to compute $y$ the relative value of the squared cluster sizes with respect to the squared number of opinions.

$$
y=\frac{\sum_{i=1}^{n} s_{i}^{2}}{\left(\sum_{i=1}^{n} s_{i}\right)^{2}}
$$

For $m$ clusters of equal size, one would have $y=1 / m$. The smaller $y$, the more important is the dispersion in opinions. Figure 8 shows the increase of the dispersion index $y$ with $n$ ( $n-1$ is the initial "subjective" weight of agent's own opinion).

The time pattern of thresholds appearing as green bands on Figure 9 gives us some insight on these effects. Because opinion exchange decays variance by an approximately constant factor close to $\alpha$, each individual green band corresponds to a given number of opinion exchanges experienced by the agents: the upper band corresponds to the variance after one exchange, the second upper to two exchanges, and so on. The horizontal width of a band corresponds to the fact that different agents are experiencing the same number of updates at different times: rough evaluations made on Figure 9 show that most agents have their first exchange between time 0 and 4000, and their fifth exchange between 1000 and 12,000.

When the decrease of threshold and the clustering of opinions is fast, those agents that are not sampled early

\section{FIGURE 9}

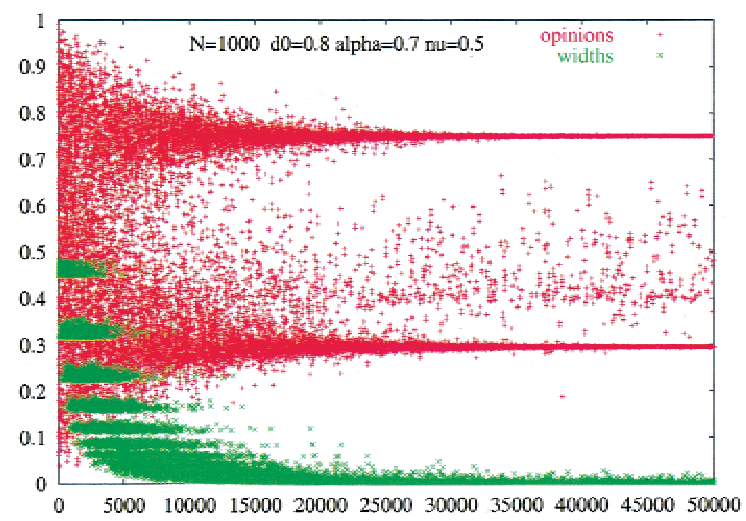

Time chart of opinions and thresholds (for constant $\alpha=0.7 d(0)$ $=0.4 v=0.5 \mathrm{~N}=1000)$. Red + represent opinions and green $\times$ represent thresholds.

enough and/or not paired with close enough agents can be left over from the clustering process. When they are sampled later, they might be too far from the other agents to get involved into opinion adjustment. The effect gets important when convergence is fast, i.e., when $n$ and $\alpha$ are small.

Let us note that these agents in the minority have larger uncertainty and are more "open to discussion" than those in the mainstream, in contrast with the common view that eccentrics are opinionated!

The results of the dynamics are even more dispersed for lower values of $\alpha$. In this regime, corresponding to "insecure agents" who do not value their own opinion more than those of other agents, we observe more clusters which importance and localization depend on the random sampling of interacting agents and are thus harder to predict than in the other regime with a small number of big clusters.

Using a physical metaphor, clustering in the small $\alpha$ regime resembles fast quenching to a frozen configuration, thus maintaining many "defects" (e.g., here the outliers), whereas in the opposite large $\alpha$ regime it resembles slow annealing (with suppression of defects).

\section{VECTOR OPINIONS}

\subsection{The Model}

Another subject for investigation is vectors of opinions. Usually people have opinions on different subjects, which can be represented by vectors of opinions. In accordance with our previous hypotheses, we suppose that one agent interacts concerning different subjects with another agent according to some distance with the other agent's vector of opinions. In order to simplify the model, we revert to binary opinions. An agent is characterized by a vector of $m$ binary 
opinions about the complete set of $m$ subjects, shared by all agents. We use the notion of Hamming distance between binary opinion vectors (the Hamming distance between two binary opinion vectors is the number of different bits between the two vectors). Here, we only treat the case of complete mixing; any pair of agents might interact and adjust opinions according to how many opinions they share. (The bit string model shares some resemblance with Axelrod's model of disseminating culture [23] based on adjustment of cultures as sets of vectors of integer variables characterising agents on a square lattice.) The adjustment process occurs when agents agree on at least $m-d$ subjects (i.e., they disagree on $d-1$ or fewer subjects). The rules for adjustment are as follows: when opinions on a subject differ, one agent (randomly selected from the pair) is convinced by the other agent with probability $\mu$. Obviously this model has connections with population genetics in the presence of sexual recombination when reproduction only occurs if genome distance is smaller than a given threshold. Such a dynamics results in the emergence of species (see Higgs and Derrida [28]). We are again interested in the clustering of opinion vectors. In fact clusters of opinions here play the same role as biological species in evolution.

\subsection{RESULTS}

We observed once again that $\mu$ and $N$ only modify convergence times toward equilibrium; the most influential factors are threshold $d$ and $m$ the number of subjects under discussion. Most simulations were done for $m=13$. For $N=$ 1000 , convergence times are of the order of 10 million pair iterations. For $m=13$ :

- When $d>3$, convergence toward a single opinion (consensus) is observed (with the exception of one or two outliers for the lower values of $d$ ).

- For $d=3$, one observes from 2 to 7 significant peaks (with a population larger than $1 \%$ ) plus some isolated opinions.

- For $d=2$ a large number (around 500) of small clusters is observed.

The same kind of results are obtained with other values of $m$ : two regimes, uniformity of opinions for larger $d$ values and extreme diversity for smaller $d$ values, are separated by one $d_{c}$ value for which a small number of clusters is observed (e.g., for $m=21, d_{c}=5 . d_{c}$ seems to scale in proportion with $m$ ).

Figure 10 represents these populations of the different clusters at equilibrium (iteration time was 12,000,000) in a log-log plot according to their rank-order of size. No scaling law is obvious from these plots, but we observe the strong qualitative difference in decay rates for various thresholds $d$.

\section{CONCLUSION}

The main lesson from this set of simulations is that opinion exchanges restricted by a small proximity threshold result

\section{FIQURE 10}

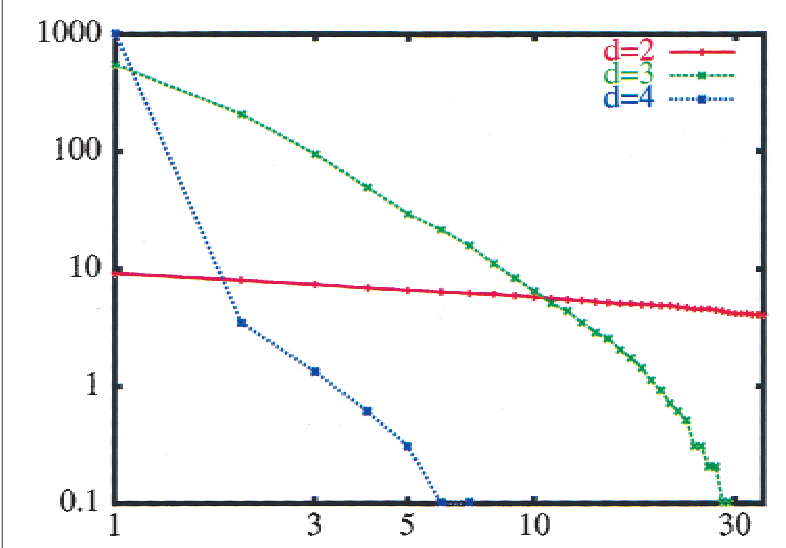

Log-log plot of average populations of clusters of opinions arranged by decreasing order for $N=1000$ agents $(\mu=1)$.

into clustering of opinions as opposed to consensus in the absence of threshold or for large thresholds.

When one gets more specific, some differences appear between the case of continuous opinions and binary strings:

- Binary strings display an abrupt phase transition from consensus to a large multiplicity of clusters when $d$ decreases.

- By contrast, for continuous opinions, the change in the number of attractors as a function of threshold is graded. The $1 / 2 \mathrm{~d}$ rule predicts the outcome of the dynamics in the simplest cases, but it also provides some qualitative insight for the case of threshold dynamics.

When we introduced dynamics on thresholds on the basis that agents interpret opinion exchange as sampling a distribution of opinions, a surprising result was that the nature of opinion and threshold updating only changes the scaling law for the time of convergence; rather, the important parameter which determines the distribution of attractors is $\alpha$ the initial self-confidence of the agents.

On the other hand, what we have shown here is that clustering of opinions does not automatically imply as a cause conflicting interests or more simply a diversity of interests and opportunities. In other words, clustering of opinions might result from the history of agents without any structural diversity.

The formal model was introduced to represent continuous opinion dynamics among groups of farmers facing uncertainties concerning the economic and/or social value of a choice; its possible range of application goes far beyond technological change issues. Obviously what we said about opinions sometimes applies to beliefs. The binary string dynamics, e.g., is an interesting approach to political debates. 
The continuous opinion dynamics can also bring some insight on the emergence of discrete coding of continuous variables, e.g., the emergence of lexicon (how big is big?).

\section{ACKNOWLEDGMENTS}

We thank David Neau and the members of the IMAGES FAIR project, Edmund Chattoe, Nils Ferrand, and Nigel Gilbert for helpful discussions. G.W. benefited at different stages in the project from discussions with Sam Bowles, Winslow Farell, and John Padgett at the Santa Fe Institute, whom we thank for its hospitality. We also thank Rainer Hegselmann for pointing us the references to the "consensus" literature. This study has been carried out with financial support from the Commission of the European Communities, Agriculture and Fisheries (FAIR) Specific RTD program, CT96-2092, "Improving Agri-Environmental Policies: A Simulation Approach to the Role of the Cognitive Properties of Farmers and Institutions." It does not necessarily reflect its views and in no way anticipates the Commission's future policy in this area.

\section{REFERENCES}

1. Images Project (1997), FAIR 3 CT 2092, http://wwwlisc.clermont.cemagref.fr//magesProject/.

2. Buttel, F.H.; Larson, O.F.; and Gillespie, G.W. The Sociology of Agriculture, Greenwood Press, Westport, CT, 1990.

3. Deffuant, G. Final report of project FAIR 3 CT 2092. Improving Agri-environmental Policies: A Simulation Approach to the Cognitive Properties of Farmers and Institutions. http://wwwlisc.clermont.cemagref.fr/ImagesProject/freport.pdf. 2001, pp 56-69.

4. Granovetter, M. Threshold models of collective behavior. Am J Soc 1978, 83, 1360-1380.

5. Valente, T.W. Network Models of the Diffusion of Innovation. Hampton Press Inc., Cresskill: NJ, 1995.

6. Föllmer, H. Random economies with many interacting agents. J Math Econ 1974, 1/1, 51-62.

7. Arthur, B.W. Increasing Returns and Path Dependence in the Economy. University of Michigan Press: Ann Arbor, MI, 1994.

8. Galam, S.; Gefen, Y.; Shapir, Y. Sociophysics: A mean behavior model for the process of strike. Math J Soc 1982, 9, 1-13.

9. Galam, S.; Moscovici, S. Towards a theory of collective phenomena: consensus and attitude changes in groups, Eur J Soc Psych 1991, $21,49-74$.

10. Latané, B.; Nowak, A. self-organizing social systems: Necessary and sufficient conditions for the emergence of clustering, consolidation and continuing diversity. In: Progress in Communication Sciences. Barnett, G.A.; Boster, F.J., Eds.; 1997, pp. 43-74.

11. Orléan, A. Bayesian interactions and collective dynamics of opinions: Herd behavior and mimetic contagion. J Econ Behav Org 1995, $28,257-274$.

12. Weisbuch, G.; Boudjema, G. Dynamical aspects in the adoption of agri-environmental measures. Adv Complex Systems 1999, 2, 11-36.

13. Stone M. The opinion pool. Ann Math Stat 1961, 32, 1339-1342.

14. Chatterjee, S; Seneta, E. Towards consensus: Some convergence theorems on repeated averaging. J Appl Prob 1977, 14, 89-97.

15. Cohen, J.E.; Hajnal, J.; Newman, C.M. Approaching consensus can be delicate when positions harden. Stochastic Processes Appl 1986, 22, 315-322.

16. Laslier, J.F. Diffusion d'information et évaluations séquentielles. Econ Appl 1989, 42-3, 155-170.

17. Krause, U. A discrete non-linear and non-autonomous model of consensus formation. In: Communications in Difference Equations. Elaydi et al., Ed.; Gordon and Breach: Amsterdam, 2000.

18. Hegselmann, R.; Krause, U. Opinion formation under bounded confidence. Proceedings of the Simsoc5 Conference. J Artific Soc Soc Simul 2002. Forthcoming.

19. Petty, R.E.; Cacioppo, J.T. The Elaboration likelihood model of persuasion In: Advances in Experimental Social Psychology, Vol. 19. Berkowitz, L. Ed.; Academic Press: New York, 1986; pp 123-205.

20. Deutsch, M.; Gerard, H.B. A study of normative and informationnal social influences upon individual judgement. J Abnormal Soc Psych 1955, 51, 629-636.

21. Tesser T. Self generated attitudes towards change. Adv Exp Soc Psychol 1978, 11, 289-338.

22. Wood, W. Retrieval of attitude relevant observation from memory: effects on susceptibility to persuasion and on intrinsic motivation. J Pers Soc Psychol 1982, 42, 798-810.

23. Axelrod R. Disseminating cultures. In: The Complexity of Cooperation; Axelrod R., Ed.; Princeton University Press: Princeton, NJ, 1997; pp $145-177$

24. Deffuant, G.; Neau, D.; Amblard, F.; Weisbuch, G. Mixing beliefs among interacting agents. Adv Complex Systems 2000, 3, 87-98.

25. Weisbuch, G.; Deffuant, D.; Amblard, F.; Nadal, J.P. (2001) Interacting Agents and Continuous Opinions Dynamics, Cond-mat/0111494 available at Xxx.lanl.gov and Santa Fe Institute Working Paper 01-11-072.

26. Neau, D. Révisions des croyances dans un systéme d'agents en interaction, rapport d'option de l'école polytechnique, available at http:// www.lps.ens.fr/ weisbuch/rapneau.ps. 2000.

27. Stauffer, D.; Aharony, A. Introduction to Percolation Theory. Taylor and Francis: London, 1994.

28. Higgs, P.G.; Derrida, B. Stochastic models for species formation in evolving populations. J Phys A Math Gen 1991, 24, 985-991. 